\title{
Influence of periodic table in designing solid-state metal chalcogenides for thermoelectric energy conversion
}

\author{
EKASHMI RATHORE ${ }^{\mathrm{a}}$, MOINAK DUTTA $^{\mathrm{a}}$ and KANISHKA BISWAS ${ }^{\mathrm{a}, \mathrm{b}, *}$ (D) \\ ${ }^{a}$ New Chemistry Unit, Jawaharlal Nehru Centre for Advanced Scientific Research (JNCASR), Jakkur P.O., \\ Bangalore 560 064, Karnataka, India \\ ${ }^{\mathrm{b}}$ School of Advanced Materials and International Centre for Materials Science, Jawaharlal Nehru Centre for \\ Advanced Scientific Research (JNCASR), Jakkur P.O., Bangalore 560 064, Karnataka, India \\ E-mail: kanishka@jncasr.ac.in \\ Ekashmi Rathore and Moinak Dutta have contributed equally to this work.
}

MS received 6 September 2019; revised 22 October 2019; accepted 23 October 2019; published online 3 December 2019

\begin{abstract}
With the apparent burgeoning energy crisis, alternative sources of energies are in a greater need for a sustainable future. Thermoelectrics which can convert waste heat arising from industries, power plants and automobiles into a usable form, electricity; have the potential to be a game-changer in this critical energy shortage. The efficiency of thermoelectric materials which is given by the figure of merit is tricky to manipulate due to the complicated interrelationships of its parameters. But with proper understanding of a material and with the aid of periodic table, one can manoeuvre the shortcomings which hinder its efficiency. In this perspective, we discuss how the properties of materials can be manipulated just by understanding the elements of the periodic table and how each element in their respective position in the periodic table influences the outcome of high performing thermoelectric material.
\end{abstract}

Keywords. Periodic trends; Metal chalcogenides; Thermoelectrics; Chemical bonding; Solid state chemistry.

\section{Introduction}

The growing demand for energy has given rise to a tremendous scope for research in thermoelectrics. Thermoelectric materials can convert waste heat into electricity. High thermoelectric (TE) performance is observed in materials with high Seebeck coefficient, $S$, low electrical resistivity, $\rho$ (high electrical conductivity, $\sigma$ ) and low thermal conductivity, $\kappa_{t o t}{ }^{1-5}$ The thermoelectric figure of merit couples these necessary attributes of good TE material into one factor as given by

$Z T=\frac{\sigma S^{2} T}{\left(\kappa_{\text {ele }}+\kappa_{\text {lat }}\right)}$

where $\kappa_{\text {ele }}$ and $\kappa_{\text {lat }}$ correspond to electrical and lattice contributions respectively, of the total thermal conductivity, $\kappa_{\text {tot }}$. These physical properties are strongly interdependent on each other and thus makes it challenging to design high-performance TE materials. To understand the fundamental material

*For correspondence properties, assuming carrier concentration, a more prominent term called the TE quality factor, $\beta$ is preferred. ${ }^{1}$

$\beta=\frac{N_{v}}{m_{i}^{*} \kappa_{l a t}}$

Large valley degeneracy, $N_{v}$ which essentially relates to the number of similar energy states around conduction band minima or valence band maxima, contributes to a large number of conduction pathways for charge carriers for electronic transport and low inertial effective mass, $m_{i}^{*}$ along the direction of conduction pathway. Effective mass is defined as the relative mass of the charge carriers with respect to the mass of free electron. Thus, effective mass being closer to the mass of free electron means the electrical conduction is much greater and can hence improve the mobility $\left(\mu=e \tau / m_{i}^{*}\right)$. For single valley, $m_{\text {band }}^{*}=m_{i}^{*}$ and the contribution of $m_{i}^{*}$ is related to density of states effective mass $m_{D O S}^{*}$ via $m_{\text {band }}^{*}$ as ${ }^{5}$

$m_{D O S=}^{*} m *_{\text {band }} N_{v}^{2 / 3}$ 
High Seebeck coefficient can either be due to large $m_{\text {band }}^{*}$ or large $N_{v}$. However, large $m_{\text {band }}^{*}$ increases scattering and decrease the mobility of electrons as it is related to $m_{i}^{*}$.

The band gap between the valence and conduction band plays an important role in TE performance of a material. When the temperature increases, the electronic carriers are thermally excited across the bandgap and the minority carriers start to neutralize the majority carriers, leading to origin of low thermo-power and high thermal conductivity due to bipolar conduction. Thus, bandgap regulates the maximum suitable operating temperature and figure of merit of TE material. ${ }^{1,6}$ Figure 1 describes the band gap of a crystalline solid with hypothetical composition $\mathrm{AB}$ by Molecular Orbital Theory (MOT). The band gap, bonding energy or strength of bonding interactions, energy difference between atomic orbitals, width of valence and conduction band is given by $E_{g}, B^{*}, 2 A^{*}, W_{V B}, W_{C B}$, respectively. In simple $\mathrm{MO}$ of solid with composition $\mathrm{AB}, B^{*}$ is the subsequent energy from stabilization of molecular bonding orbitals and destabilization of antibonding orbital compared to atomic orbitals. $B^{*}$ can be expressed in terms of orbital overlap interaction, $V$ and $A^{*}$ as ${ }^{7,8}$ :

$B^{*}=\sqrt{V^{2}+A^{* 2}}-A^{*}$

Larger $A^{*}$ will lead to weaker covalent bonding energy, $B^{*} . V$ is indirectly proportional to the square of the distance, $d$ which can describe the valence shell interactions. According to Koopman's theorem, higher is the electronegativity, lower is $A^{*}{ }^{.9}$ Large difference in electronegativity $(\chi)$ between element A with small $\chi$ and $\mathrm{B}$ with large $\chi$ results in larger band gap. The orbital character of bands is also influenced by $\chi$ difference. In

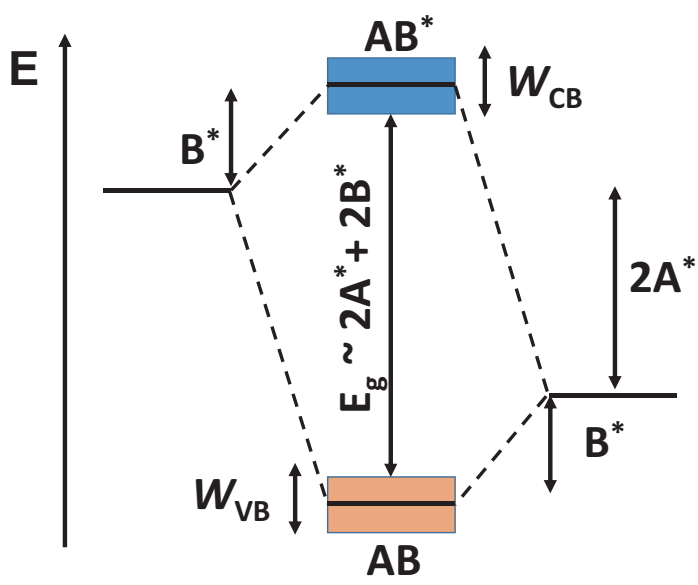

Figure 1. Molecular orbital (MO) diagram of solid with composition $\mathrm{AB}$, where $E_{g}$ is bandgap, $A^{*}$ is energy difference of atomic orbitals, $\mathrm{W}$ is the width of conduction and valence band, $B *$ is the strength of bonding interactions. ionic solids with large $A^{*}$, the valence band states will have anion $\mathrm{B}$ character and conduction band states will have cation A element character.

The electronic properties in semiconductors are governed by the width of an electronic band $W^{2}$ Figure 1 shows that increased width $W$ can lead to the reduction of the band gap and it is possible to tune the electronic properties. If $W>B^{*}+A^{*}, \mathrm{AB}$ and $\mathrm{AB}^{*}$ $\mathrm{MO}$ orbitals of $\mathrm{AB}$ will merge to form an overlapping band and result into metal or semimetal. For polar compounds $\left(A^{*} \neq 0\right), W$ tends to drop with an increase in $A^{*}$ as the overlap, $V$ becomes less effective due to the rise in the energy difference between the neighboring orbitals leading to large band gaps in ionic compounds. Smaller dispersion (narrow band) arises from factors like longer bonds, high degree of polarity or dispersed f-orbitals which have lower overlap than p- or s-interactions. Narrow band in energy space corresponds to wide parabola in $\mathrm{k}$-space, a large DOS and a high $m_{\text {band }}^{*}$ which is expected in large electronegative difference ionic compounds. ${ }^{8}$

Arguably the most important parameter in the thermoelectric figure of merit is that of thermal conductivity. The total thermal conductivity $\left(\kappa_{t o t}\right)$ is a summation of two types of heat propagators. One of the heat propagators are the free charge carriers (electron or hole), and the amount of heat propagation by them is given by $\kappa_{e l e}$; while the other is heat transport via lattice vibration (or phonons) which is denoted as $\kappa_{\text {lat }}$. Since high $\kappa_{\text {ele }}$ is a resultant of high $\sigma$ by virtue of their relation using Wiedemann-Franz law (i.e., $\kappa_{\text {ele }}=\mathrm{L} \sigma \mathrm{T}, \mathrm{L}=$ Lorentz number), reducing $\kappa_{e l e}$ is not a popular choice and thus reducing $\kappa_{\text {lat }}$ is seen as an important avenue to achieving high performance in thermoelectrics. As a matter of fact, almost all high performing thermoelectric materials possess low thermal conductive parent compound and is often considered to be the backbone in designing superior, highly efficient thermoelectric material. ${ }^{10-12}$ Periodic table more often than not dictates how the material will transport heat and along with keen chemical intuition, one often designs compounds showing low thermal conductive materials. For example, the tellurides of $\mathrm{Ge}$ to $\mathrm{Pb}$ in group 14 (i.e., GeTe, $\mathrm{SnTe}$ and $\mathrm{PbTe}$ ), we see a decreasing trend in the thermal conductivity of the material. We have discussed the influence of periodic table in generating low thermal conductive materials in more detail in later part of our review.

Thus, we can say that the electronic and thermal transport are linked to bonding character, band gap, bonding strength, bond length, effective mass, orbital overlap and lone-pair anharmonicity; all of them can be understood by a thorough understanding of the 
elements and their position on the periodic table. In this review, we will discuss the importance that the periodic table plays in shaping the thermoelectric properties of materials.

\section{Periodic trends in electronic properties}

\subsection{Band gap}

Band gap is one of the contributing factors in determining the electrical conductivity $(\sigma)$ of a semiconductor. Generally, materials with low band gap show high $\sigma$ and decrease with increasing band gap. In case of $\mathrm{PbQ}(\mathrm{Q}=$ chalcogenides, $\mathrm{S}, \mathrm{Se}$ and $\mathrm{Te})$, the band gap for $\mathrm{PbSe}(0.27 \mathrm{eV})<\mathrm{PbTe} \quad(0.31 \mathrm{eV})<\mathrm{PbS}$ $(0.40 \mathrm{eV})$. The electrical conductivity too shows the same trend as $(\sigma(\mathrm{PbSe})>\sigma(\mathrm{PbTe})>\sigma(\mathrm{PbS})) .{ }^{13}$ For thermoelectrics, the materials should possess band gap which falls in the semi-conducting region in terms of band edge (i.e., neither very low metallic gap or high band gap as that of an insulator). Often $\sigma$ is governed by the mobility of the major carriers in the material. Carrier mobility, $\mu$ is low for bands with heavy mass $m_{\text {band }}^{*}$, leading to low electrical conductivity $(\sigma=n e \mu)$. This difference between the valence band and conduction band relies upon several factors like electronegativity, presence of stereochemical lone pairs and temperature, etc., and are known to play a crucial role in determining the electrical conductivity of a material.

\subsection{Role of electronegativity}

The difference in electronegativity can be helpful in tuning the band gaps and thereby the electronic mobility as $\mathrm{E}_{\mathrm{g}} \sim 2 A^{*}+2 B^{*}$ and $A^{*}$ depends on electronegativity. Larger is the electronegativity difference more will be band gap. For example, in $\mathrm{AgBiSe}_{2-x} \mathrm{X}_{x}$ $(\mathrm{X}=\mathrm{Cl}, \mathrm{Br}, \mathrm{I})$ the electrical conductivity for pristine $\mathrm{AgBiSe}_{2}$ is around $63 \mathrm{~S} \mathrm{~cm}^{-1}$. On doping aliovalent halogens, the band gap is seen to widen up (from $\sim 0.6$ $\mathrm{eV})$ due to higher electronegativity of halogens $\left(\chi_{\mathrm{Cl}}\right.$ (3.16) $\left.>\chi_{\mathrm{Br}}(2.96)>\chi_{\mathrm{I}}(2.66)\right)$ as compared to selenium $\left(\chi_{\mathrm{Se}}=2.55\right)$ which causes more ionic character in $\mathrm{M}-\mathrm{X}$ bond than that of M-Se bond. ${ }^{14}$ As a result, the $\sigma$ increases to $376 \mathrm{~S} \mathrm{~cm}^{-1}$ at room temperature for $4 \mathrm{~mol} \% \mathrm{Cl}$ doped $\mathrm{AgBiSe} 2$. For $4 \mathrm{~mol} \% \mathrm{Br}$ and I doped $\mathrm{AgBiSe}_{2}, \sigma$ value is $337 \mathrm{~S} \mathrm{~cm}^{-1}$ and $196 \mathrm{~S} \mathrm{~cm}^{-1}$ respectively at room temperature. ${ }^{14}$ The $\sigma$ trend for aliovalent halogen doped $\mathrm{AgBiSe}_{2}$ mirrors the electronegativity trend down the Group 17 of the periodic table (Figure 2a). Thus, proper knowledge of periodic table and their corresponding electronegativity aids in realizing high thermoelectric performance in n-type $\mathrm{AgBiSe}_{2}{ }^{14}$ Similarly for PbSe, ongoing down Group 13 , (i.e., $\mathrm{B}, \mathrm{Al}, \mathrm{Ga}), \sigma$ is seen to increase from 8.72 $\left(10^{2} \mathrm{~S} \mathrm{~cm}^{-1}\right)$ in case of $\mathrm{B}$ doped $\mathrm{PbSe}$ to around 38.5 $\left(10^{2} \mathrm{~S} \mathrm{~cm}^{-1}\right)$ for Ga doped PbSe. ${ }^{15}$ Here too, the periodic trend is observed; as we go down the group, the electronegativity decreases which increases the propensity of Group 13 elements to donate excess electrons and thereby increases the carrier concentration which in turn increases the $\sigma$ of n-type PbSe.

\subsection{Effect of lone-pairs}

In the elements of group 13, 14 and 15, the lone pair of electrons play an important role in the electronic properties of thermoelectric materials. As we move down the group in $\mathrm{p}$-block elements, the $\mathrm{ns}^{2}$ lone pair experiences a greater effective nuclear force, and as a result tends to become inert in nature. In group 13, as the element becomes heavier from $\mathrm{Ga}$ to $\mathrm{Tl}$, the lone pair becomes more stabilized in $\mathrm{In}^{+}$than in $\mathrm{Ga}^{+}$. In case of group 14 and $15, \mathrm{~Pb}^{2+}$ and $\mathrm{Bi}^{3+}$ have very stable lone pair and it is difficult to form $\mathrm{Pb}^{4+}$ and $\mathrm{Bi}^{5+}$. This enormous stability of $n s^{2}$ lone pairs is due to relativistic effects which reduce the size of s-orbital and bring the electron near to nucleus. In semiconductors, containing $\mathrm{Tl}, \mathrm{Ge}, \mathrm{Sn}, \mathrm{Pb}$, $\mathrm{Sb}$ and $\mathrm{Bi}$, the stereochemical expression of $n s^{2}$ lone pair, increases the band gap as it stabilizes in energy. ${ }^{2}$ Depending upon the chalcogenides bonded to these metal ions, the lone pair is either stereochemically expressed or quenched. Down the group, there is a tendency to quench the lone pair and adopt high local symmetry. This concept can be realized in many examples and these are listed in Table $1 . \mathrm{In}_{2} \mathrm{~S}_{3}, \mathrm{SnSe}$, $\mathrm{Sb}_{2} \mathrm{Se}_{3}, \mathrm{GeS}$ the lone pair stereochemically expressed, causes strong distortion and lowering of structural symmetry which lowers the energy of the orbitals significantly and thereby increasing the bandgap. Thus, the lone pairs become the contributing factor in estimating the band gap of a system and its influence on the electrical conductivity.

\subsection{Seebeck coefficient}

Seebeck coefficient is related to electrical conductivity by Mott equation (Eq. 5). $S$ is the measure of variation of $\sigma(E)$ above and below the Fermi surfaces. $\sigma(E)$ is proportional to the DOS at $\mathrm{E}$ if the scattering of electrons is independent of energy. A larger slope (d $\ln \sigma(E) / \mathrm{d} E$ ) where DOS changes rapidly, will contribute to larger thermopower. ${ }^{1}$ 

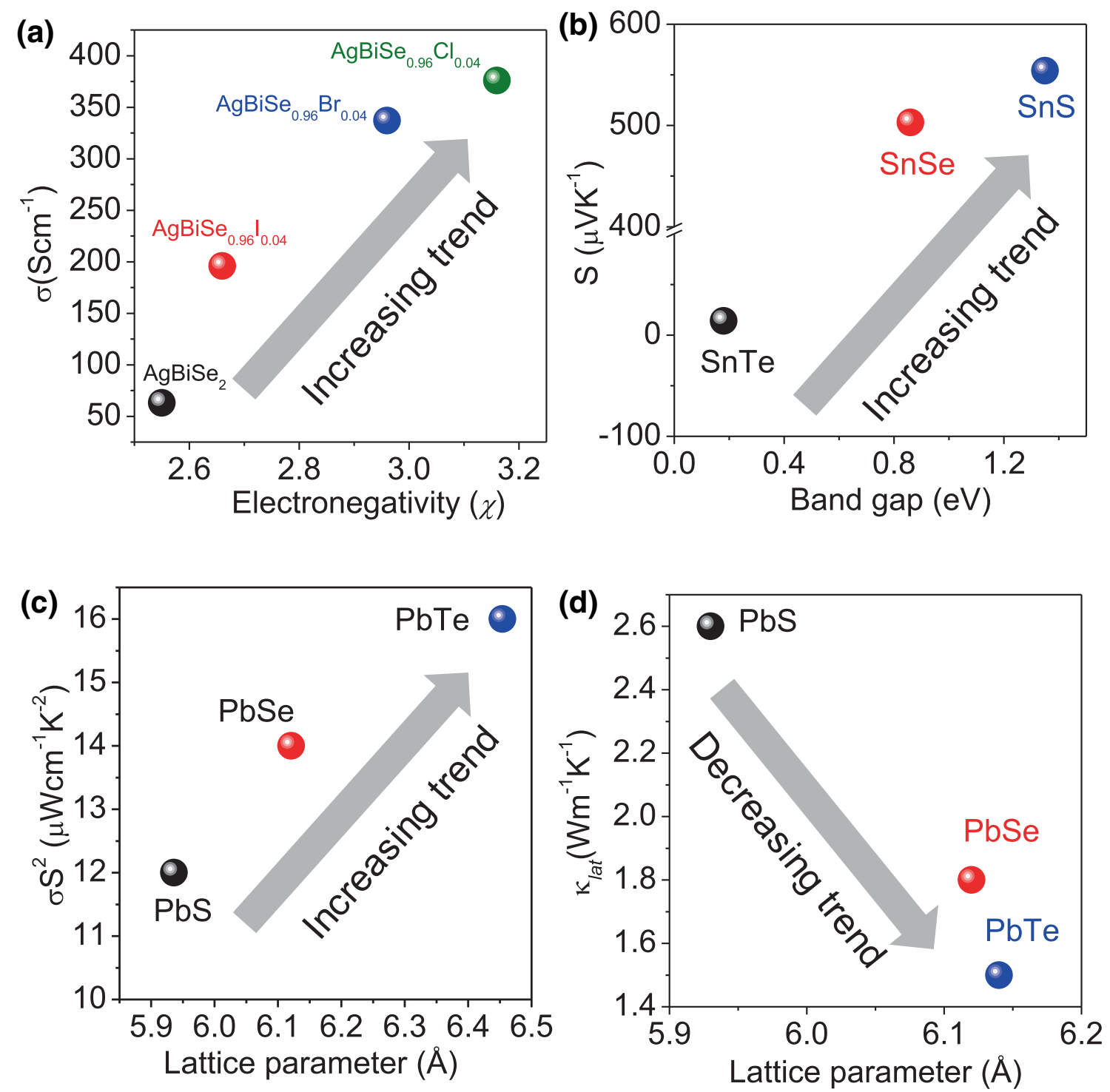

Figure 2. Periodic trends of (a) electrical conductivity $(\sigma)$, (b) Seebeck coefficient $(\mathrm{S})$, (c) Power factor $\left(\sigma \mathrm{S}^{2}\right)$ and (d) lattice thermal conductivity $\left(\kappa_{\text {lat }}\right)$ for some metal chalcogenides.

Table 1. Comparison of band gaps of chalcogenide based semiconductors down the group.

\begin{tabular}{lcc}
\hline Semiconductor & Crystal structure (symmetry) & Band gap (eV) \\
\hline $\mathrm{Bi}_{2} \mathrm{~S}_{3}$ & Orthorhombic (low) & 1.30 \\
$\mathrm{Bi}_{2} \mathrm{Se}_{3}$ & Rhombohedral (high) & 0.30 \\
$\mathrm{SnSe}$ & Orthorhombic (low) & 0.80 \\
$\mathrm{SnTe}$ & Cubic (high) & 0.18 \\
$\mathrm{Sb}_{2} \mathrm{Se}_{3}$ & Orthorhombic (low) & 1.10 \\
$\mathrm{Sb}_{2} \mathrm{Te}_{3}$ & Rhombohedral (high) & 0.11 \\
$\mathrm{GeSe}_{\mathrm{GeTe}}$ & Orthorhombic (low) & 1.07 \\
\end{tabular}

$$
S=\frac{\pi}{3} \frac{k^{2} T}{e}\left[\frac{d \ln (E)}{d E}\right]_{E=E_{f}}
$$

$$
S=\frac{8 \pi^{2} k_{B}^{2} T}{3 e h^{2}} m_{D O S}^{*}\left[\frac{\pi}{3 n}\right]^{2 / 3}
$$

Also, $S$ is expressed in relation to density of state effective mass $\left(m^{*}\right)$ of the carrier by the equation below: ${ }^{16}$ and density of states is given by: 
$g(E)=\frac{\left[m_{D O S}^{*}\right]^{3 / 2} \sqrt{ } 2 E}{\hbar^{3} \pi^{2}}$

In SnQ (Q = S/Se/Te), down the Group 16, the band edge decreases, SnS having a band gap of $1.35 \mathrm{eV}^{17}$ decreases to $0.86 \mathrm{eV}$ for $\mathrm{SnSe}^{18}$ to around $0.18 \mathrm{eV}$ for SnTe. ${ }^{19}$ This band edge decrease is also seen to increase the $S$ value for SnQ. SnTe having $S$ value of $\sim 14 \mu \mathrm{VK}^{-1}$ is much lower than $\mathrm{SnSe}$ $\left(\sim 503 \mu \mathrm{VK}^{-1}\right)$ or $\mathrm{SnS}\left(554 \mu \mathrm{VK}^{-1}\right)$ at ambient temperatures and the trend is continuous and in line with the periodic table trend (Figure 2b). ${ }^{19,20}$ Thus, here also, periodic table shows a tremendous influence in monitoring the Seebeck coefficient of thermoelectric materials. It is to be noted that $\mathrm{PbQ}(\mathrm{Q}=\mathrm{S} / \mathrm{Se} / \mathrm{Te})$ does not follow the trend of decreasing band gap with increasing atomic number. Chalcogenides of $\mathrm{Sn}$ and $\mathrm{Ge}$ which resides on the same Group 14 follows the normal trend. This anomaly in PbQ may have to do with relativistic effects and an extensive spin-orbit coupling which renders it impossible to predict the band gap just from a simplified MO model and general trends.

\subsection{Power factor}

In $\mathrm{PbQ},(\mathrm{Q}=\mathrm{S}, \mathrm{Se}, \mathrm{Te})$, the room temperature power factor increases from $\sim 12 \mu \mathrm{W} \mathrm{cm}{ }^{-1} \mathrm{~K}^{-2}$ for PbS to $\sim 14 \mu \mathrm{W} \mathrm{cm}{ }^{-1} \mathrm{~K}^{-2}$ for PbSe and $\sim 16 \mu \mathrm{W} \mathrm{cm}{ }^{-1}$ $\mathrm{K}^{-2}$ for PbTe. This is directly related to the size of the anion going down the group and the lattice parameter (Figure 2c). ${ }^{13} \mathrm{~A}$ similar trend is observed in $\mathrm{MSe}$ $(\mathrm{M}=\mathrm{Pb}, \mathrm{Sn}, \mathrm{Ge})$ series, where the room temperature power factor increases from $\sim 1.2 \times 10^{11} \mathrm{~W} \mathrm{mK}^{-2}$ $\mathrm{s}^{-1}$ for PbSe to $\sim 4 \times 10^{11} \mathrm{~W} \mathrm{mK}^{-2} \mathrm{~s}^{-1}$ for SnSe and $\sim 8 \times 10^{11} \mathrm{~W} \mathrm{mK}^{-2} \mathrm{~s}^{-1}$ for GeSe due more overlapping factor. ${ }^{16}$

\section{Periodic trends in thermal conductivity}

Achieving a high thermoelectric figure of merit often relies on materials having low $\kappa_{\text {lat. }}{ }^{3,10,21}$ Often an overlooked parameter in the figure of merit equation, but with recent developments in the field of thermoelectrics $\kappa_{l a t}$ is often regarded as the defining parameter to achieve superior thermoelectric performance. $\mathrm{SnSe}$, for example, has achieved ultrahigh ZT $(>2.5)$ in both $\mathrm{n}$ - and p-type conduction owing to the impressively low $\kappa_{\text {lat }}$ values $\left(\kappa_{\text {lat }} 0.2-0.3 \mathrm{Wm}^{-1} \mathrm{~K}^{-1}\right.$ at $773 \mathrm{~K}$ ) in both single-crystalline and polycrystalline forms. ${ }^{10,21-23}$ The most extensive work on achieving $\kappa_{\text {lat }}$ in materials is via introducing micro and/or nanostructuring in the matrix, ${ }^{3,11}$ while recent scientific explorations focus on looking at the intrinsic bonding inhomogeneity ${ }^{24-26}$ and enhanced anharmonicity $^{27,28}$ to lower the phonon propagation in materials. Here, however, we will focus on the influence of chemical bonds and nature of phonon propagation trending with periodic table and will develop an understanding of how the periodic table shapes up the $\kappa_{\text {lat }}$ of certain high performing materials.

Kinetic theory predicts that the $\kappa_{l a t}$ of material can be quantified approximately using the equation $\kappa_{\text {lat }}=$ $\frac{1}{3} C_{v} v_{m} l$; where $C_{v}$ is the specific heat at constant volume, $l$ is the mean free path length for phonons and $v_{m}$ is the mean speed of sound. $v_{m}$ depicts the speed of phonon waves propagating through a lattice and is given by $v_{m} \sim \sqrt{k / M}$, where $k$ is the spring constant and $\mathrm{M}$ is its molar mass. Thus, high bond strength results in high spring constant and eventually a higher $\kappa_{l a t}$ as envisaged earlier by Ioffe and Spitzer. ${ }^{1}$

$\mathrm{Pb}$ based chalcogenides, $\mathrm{PbQ}$ (i.e., $\mathrm{Q}=\mathrm{Te}, \mathrm{Se}$ and S) adopt a rock-salt type structure, on going down Group 16, the $v_{m}$ decreases. As a result, the $\kappa_{\text {lat }}$ trend also depicts this periodic trend of decreasing $v_{m}$. $\mathrm{PbS}$ have a $\kappa_{\text {lat }}$ of $\sim 2.6 \mathrm{Wm}^{-1} \mathrm{~K}^{-1}$ at room temperature and going down the group it decreases to $\sim 1.8$ $\mathrm{Wm}^{-1} \mathrm{~K}^{-1}$ and $\sim 1.5 \mathrm{Wm}^{-1} \mathrm{~K}^{-1}$ for $\mathrm{PbSe}$ and $\mathrm{PbTe}$, respectively at room temperature (Figure 2d). ${ }^{13}$ However, for $\mathrm{Ge}$ based chalcogenides, we don't observe such trend in $\kappa_{\text {lat }}$ values. GeTe have a $\kappa_{\text {lat }}$ value of $\sim 3.0 \mathrm{Wm}^{-1} \mathrm{~K}^{-1}$ at room temperature, while GeSe possesses much lower $\kappa_{\text {lat }}$ values of 1.76 $\mathrm{Wm}^{-1} \mathrm{~K}^{-1}$ at room temperature. GeS possesses slightly higher $\kappa_{l a t}$ than that of GeSe but lower than GeTe. $^{29}$ The difference in trend here can be understood in terms of understanding the structure and the energy of the valence electrons of Ge $4 p$ and $4 s$ orbitals relative to the chalcogenides $n p$ orbitals (Figure 3). On going down the Group 16, the electronegativity of the $\mathrm{Q}$ decreases (i.e., $\chi_{\mathrm{S}}$ $\left.(2.58)>\chi_{\mathrm{Se}}(2.55)>\chi_{\mathrm{Te}}(2.1)\right)$ and as a result, the energy of the $p$ orbitals on the Q increases (Figure 3). For GeTe, the difference in energy between $4 s$ of Ge and $5 p$ of Te is high enough for any significant overlap between them, and hence remains in relatively higher symmetry as compared to GeSe and GeS. Se and S whose $4 p$ and $3 p$, respectively are lower in energy can form significant bonding interaction with the $\mathrm{Ge}$ $4 s$ and as a result the lone pairs on Ge stereochemically express themselves, which drive the structure to a lower symmetric layered structure (Figure 3). These stereochemically active lone pairs induce lattice anharmonicity which in turn lowers the $\kappa_{\text {lat }}$ of GeSe and $\mathrm{GeS}$ as compared to GeTe. For SnQ, Sn-based 


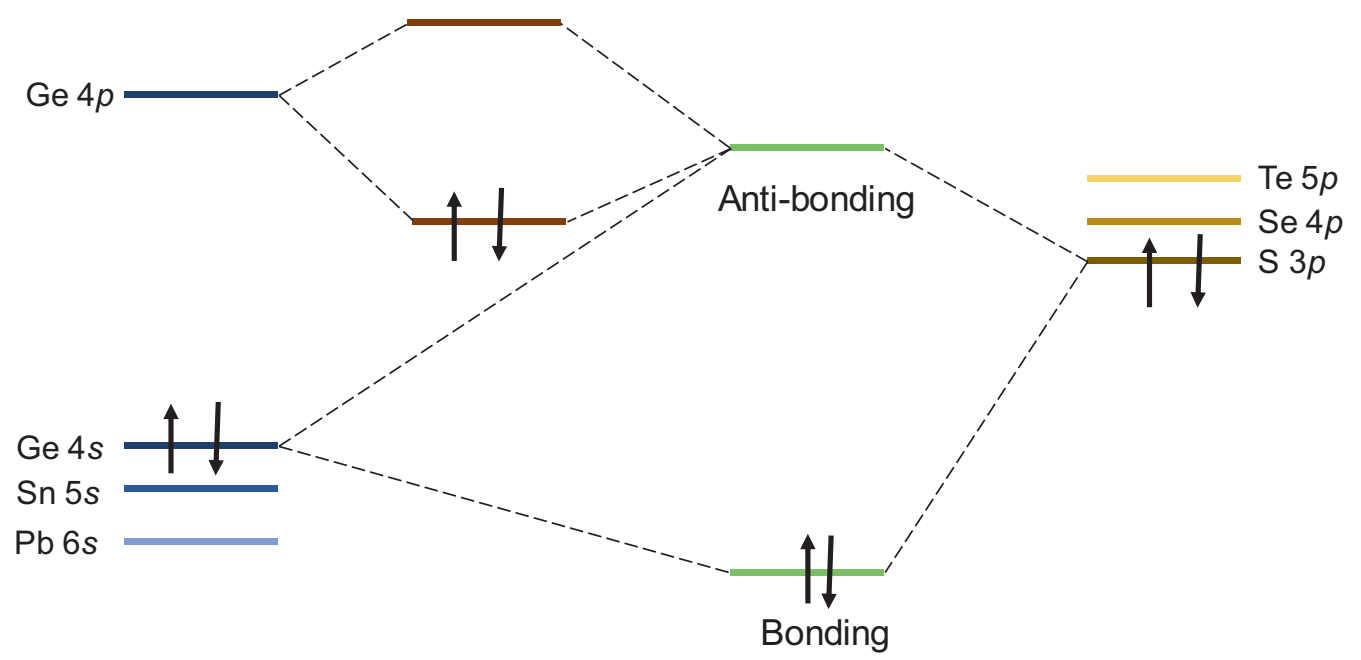

Figure 3. Modified MO diagram showing the interaction of $n s$ orbitals of $\mathrm{Ge}(\mathrm{Sn}, \mathrm{Pb})$ with $n p$ orbitals of $\mathrm{S}(\mathrm{Se}, \mathrm{Te})$. The corresponding interaction results in an opening of band gap as the anti-bonding orbital interacts with $4 p$ orbital of Ge.

chalcogenides show similar trend as that of GeQ. SnTe has $\kappa_{\text {lat }}$ of $\sim 3.1 \mathrm{Wm}^{-1} \mathrm{~K}^{-1}$ at room temperature which is significantly higher than that of $\mathrm{SnSe}$ $\left(0.46 \mathrm{Wm}^{-1} \mathrm{~K}^{-1}\right)$ and $\mathrm{SnS}\left(\sim 0.9 \mathrm{Wm}^{-1} \mathrm{~K}^{-1}\right)$ at room temperature. ${ }^{19}$ The reason for such trend is in line with the GeQ. However, SnTe at room temperature procures rock-salt structure contrary to GeTe which is rhombohedral in nature. The reason being the different energy states of $4 s$ for Ge and $5 s$ in case of $\mathrm{Sn}$. The energy gap between $5 s$ of $\mathrm{Sn}$ and $5 p$ of Te is too much to have any meaningful interaction and thus the stereochemical nature of $5 s^{2}$ lone pairs are suppressed and the structural distortion does not take place (Figure 3), unlike GeTe. Comparing MTe compounds now $(\mathrm{M}=\mathrm{Ge}, \mathrm{Sn}, \mathrm{Pb})$, we observe that the structure changes from rhombohedral in case of GeTe, to cubic in case of SnTe and PbTe. This is a direct result of the amount of interaction between $n s$ orbitals of $\mathrm{M}$ and $5 p$ of Te. The trend here directly relates to the the interaction between anion $p$ and cation $s$ and their corresponding distortion from the rocksalt structure. ${ }^{30} \mathrm{PbQ}$, on the other hand, all resides as rocksalt type structure. That is due to the relativistic effect on $6 s^{2}$ lone pairs which stabilizes them to a much greater extent. ${ }^{31}$ Although in case of $\mathrm{PbQ}$ and $\mathrm{SnTe}$, the bonding interaction among them is not significant, there remains a substantial quantity of $s$ character in the anti-bonding state. As a result, the structure although attains a globally cubic structure, but locally distorts due to trivial but finite contribution from the $n s^{2}$ lone pairs on $\mathrm{Sn}$ and $\mathrm{Pb}$ which is observed from the local structure determining technique Pair Distribution Function. ${ }^{32,33}$ This results in local off-centring of cation in these materials (i.e., PbQ and $\mathrm{SnTe}$ ) which lowers their thermal conductivity from the ideal one and also gives rise to lone pair induced anharmonicity in the lattice which is also responsible for scattering of phonons in the lattice and lowering of $\kappa_{\text {lat }}$. Thus, periodic table enables a chemist to logically intuit the outcome of $\kappa_{\text {lat }}$ in a material and thereby enhances the probability for furnishing high performing thermoelectric materials.

\section{Figure of merit}

The thermoelectric figure of merit (ZT) which accounts for both the aforementioned properties (i.e., electronic and thermal) leads to efficient values for heavier elements (i.e., $\mathrm{Pb}$ and $\mathrm{Te}$ ). As seen from the above discussions, this is how the periodic properties optimize the TE parameters which show PbTe with high electronic properties, yet surprisingly low thermal conductivity. The factors influencing such high efficiency in PbTe directly arise from the optimal band gap of $\sim 0.3 \mathrm{eV}$ and low $\kappa_{\text {lat }}$ of $1.5 \mathrm{Wm}^{-1} \mathrm{~K}^{-1}$. As a result, $\mathrm{PbTe}$ shows unmatched thermoelectric ZT values (ZT $\sim 2$.2) with very high device efficiencies using appropriate dopants. ${ }^{3,34-36}$ The use of dopants to stimulate higher thermoelectric properties are directly governed by the position in the periodic table and one can easily engineer the properties influencing the $\mathrm{ZT}$ value by proper intuition and knowledge of the periodic table.

\section{Cost of materials}

Apart from the low thermal conductivity and good thermoelectric performance, there is also a need for earth-abundant, non-toxic, and low-cost constituent 
elements. The leading advanced thermoelectric materials are mainly based on $\mathrm{Pb}, \mathrm{Ge}, \mathrm{Se}, \mathrm{Te}$. There is a need to explore attractive potential alternatives due to the toxicity of $\mathrm{Pb}$, extreme scarcity of $\mathrm{Te}$, cost issues related to Ge. Extensive research on inexpensive sulfur which has long-price stability and abundance is required. Down the group, as the element becomes heavier, it becomes less abundant and costly.

\section{Conclusions}

In conclusion, the advances in thermoelectric materials have come a long way since its inception in the late 1940s. A plethora of new techniques like the formation of resonant level, band convergence, formation of hierarchical nano/meso-architectures skyrocketed the development in this field. A ZT $>2$ which was inconceivable in the past have been achieved and now with the aid of theoretical guidance and materials genome project, a vast plethora of new materials with potential to furnish efficient thermoelectric materials are being generated. But, even all these new techniques rely on the periodic table to dictate their performance. Without the periodic table, attaining such high ZT values would not have been possible. It guides a scientist to look into the right direction and with proper knowledge and keen understanding of the periodic properties, one can easily attain a right dopant to improve on the already known thermoelectric materials or even better, furnish a new and high performing one. But one must keep in mind that even with all the prowess of the periodic table, it alone is not enough to cater to the scientific query for high performing thermoelectric materials. Integrating the knowledge obtained from periodic table with theoretical calculations and the chemical intuition to apply the required techniques like nano-structuring will pave a long way to develop materials having superior thermoelectric performances.

\section{Acknowledgements}

M.D. thanks the University Grants Commission (UGC) for funding. We thank SERB and DST for supporting the thermoelectric program in our group.

\section{References}

1. Zeier W G, Zevalkink A, Gibbs Z M, Hautier G, Kanatzidis M G and Snyder G J 2016 Thinking like a chemist: Intuition in thermoelectric materials Angew. Chem. Int. Ed. 556826
2. Zhao L-D, Dravid V P and Kanatzidis M G 2014 The panoscopic approach to high performance thermoelectrics Energy Environ. Sci. 7251

3. Biswas K, He J, Blum I D, Wu C-I, Hogan T P, Seidman D N, Dravid V P and Kanatzidis M G 2012 Highperformance bulk thermoelectrics with all-scale hierarchical architectures Nature 489414

4. J R, Chung D Y and Kanatzidis M G 2009 New and old concepts in thermoelectric materials Angew. Chem. Int. Ed. 488616

5. Tan G, Zhao L-D and Kanatzidis M G 2016 Rationally designing high-performance bulk thermoelectric materials Chem. Rev. 11612123

6. Gibbs Z M, Kim H, Wang H, White R L, Drymiotis F, Kaviany M and Snyder G J 2013 Temperature dependent band gap in $\mathrm{PbX}(\mathrm{X}=\mathrm{S}, \mathrm{Se}, \mathrm{Te})$ Appl. Phys. Lett. 103262109

7. Harrison W A 1989 Tight-binding theory of molecules and solids Pure Appl. Chem. 612161

8. Rohrer G S 2001 Structure and bonding in crystalline materials (Cambridge: Cambridge University Press)

9. Koopmans T 1934 Ãœber die Zuordnung von Wellenfunktionen und Eigenwerten zu den Einzelnen Elektronen Eines Atoms Physica 1104

10. Zhao L-D, Lo S-H, Zhang Y, Sun H, Tan G, Uher C, Wolverton C, Dravid V P and Kanatzidis M G 2014 Ultralow thermal conductivity and high thermoelectric figure of merit in SnSe crystals Nature $\mathbf{5 0 8} 373$

11. Samanta M and Biswas K 2017 Low Thermal conductivity and high thermoelectric performance in $(\mathrm{GeTe})_{1-2 \mathrm{x}}(\mathrm{GeSe})_{\mathrm{x}}(\mathrm{GeS})_{\mathrm{x}}$ : competition between solid solution and phase separation J. Am. Chem. Soc. 139 9382

12. Zhu T, Liu Y, Fu C, Heremans J P, Snyder J G and Zhao X 2017 Compromise and synergy in high-efficiency thermoelectric materials Adv. Mater. 291605884

13. Pei Y-L and Liu Y 2012 Electrical and thermal transport properties of $\mathrm{Pb}$-based chalcogenides: $\mathrm{PbTe}, \mathrm{PbSe}$, and $\mathrm{PbS}$ J. Alloys Compd. $\mathbf{5 1 4} 40$

14. Guin S N, Srihari V and Biswas K 2015 Promising thermoelectric performance in n-type $\mathrm{AgBiSe}_{2}$ : effect of aliovalent anion doping J. Mater. Chem. A 3648

15. Zhang Q, Cao F, Lukas K, Liu W, Esfarjani K, Opeil C, Broido D, Parker D, Singh D J, Chen G and Ren Z 2012 Study of the thermoelectric properties of lead selenide doped with boron, gallium, indium, or thallium J. Am. Chem. Soc. 13417731

16. Ding G, Li J and Gao G 2015 Band structure engineering of multiple band degeneracy for enhanced thermoelectric power factors in MTe and MSe $(\mathrm{M}=\mathrm{Pb}, \mathrm{Sn}, \mathrm{Ge})$ RSC Adv. 591974

17. Albers W, Haas C, Vink H J and Wasscher J D 1961 Investigations on SnS J. Appl. Phys. 322220

18. Zhou Y and Zhao L-D 2017 Promising Thermoelectric Bulk Materials with 2D Structures Adv. Mater. 291702676

19. Banik A, Roychowdhury S and Biswas K 2018 The journey of tin chalcogenides towards high-performance thermoelectrics and topological materials Chem. Commun. 546573

20. Han Y-M, Zhao J, Zhou M, Jiang X-X, Leng H-Q and Li L-F 2015 Thermoelectric performance of SnS and SnS-SnSe solid solution J. Mater. Chem. A 34555 
21. Chandra S and Biswas K 2019 Realization of high thermoelectric figure of merit in solution synthesized 2D SnSe nanoplates via Ge Alloying J. Am. Chem. Soc. 1416141

22. Chang C, Wu M, He D, Pei Y, Wu C-F, Wu X, Yu H, Zhu F, Wang K, Chen Y, Huang L, Li J-F, He J and Zhao L-D 2018 3D charge and 2D phonon transports leading to high out-of-plane $Z T$ in n-type SnSe crystals Science $\mathbf{3 6 0} 778$

23. Lee Y K, Luo Z, Cho S P, Kanatzidis M G and Chung I 2019 Surface oxide removal for polycrystalline SnSe reveals near-single-crystal thermoelectric performance Joule 3719

24. Dutta M, Pal K, Waghmare U V and Biswas K 2019 Bonding heterogeneity and lone pair induced anharmonicity resulted in ultralow thermal conductivity and promising thermoelectric properties in n-type $\mathrm{AgPbBiSe}_{3}$ Chem. Sci. 104905

25. Jana M K, Pal K, Warankar A, Mandal P, Waghmare U $\mathrm{V}$ and Biswas K 2017 Intrinsic rattler-induced low thermal conductivity in zintl type $\mathrm{TlInTe}_{2} \mathrm{~J}$. Am. Chem. Soc. 1394350

26. Samanta M, Pal K, Pal P, Waghmare U V and Biswas K 2018 localized vibrations of Bi bilayer leading to ultralow lattice thermal conductivity and high thermoelectric performance in weak topological insulator n-type BiSe J. Am. Chem. Soc. 1405866

27. Morelli D T, Jovovic V and Heremans J P 2008 Intrinsically minimal thermal conductivity in cubic I-V-VI 2 semiconductors Phys. Rev. Lett. 101035901

28. Guin S N, Chatterjee A, Negi D S, Datta R and Biswas K 2013 High thermoelectric performance in tellurium free p-type $\mathrm{AgSbSe}_{2}$ Energy Environ. Sci. 62603

29. Roychowdhury S, Samanta M, Perumal S and Biswas K 2018 Germanium chalcogenide thermoelectrics: electronic structure modulation and low lattice thermal conductivity Chem. Mater. 305799

30. Waghmare U V, Spaldin N A, Kandpal H C and Seshadri R 2003 First-principles indicators of metallicity and cation off-centricity in the IV-VI rocksalt chalcogenides of divalent $\mathrm{Ge}, \mathrm{Sn}$, and $\mathrm{Pb}$ Phys. Rev. B 67125111

31. Walsh A, Payne D J, Egdell R G and Watson G W 2011 Stereochemistry of post-transition metal oxides: revision of the classical lone pair model Chem. Soc. Rev. 404455

32. Knox K R, Bozin E S, Malliakas C D, Kanatzidis M G and Billinge S J L 2014 Local off-centering symmetry breaking in the high-temperature regime of SnTe Phys. Rev. B 89014102

33. Božin E S, Malliakas C D, Souvatzis P, Proffen T, Spaldin N A, Kanatzidis M G and Billinge S J L 2010 Entropically stabilized local dipole formation in lead chalcogenides Science 3301660

34. Biswas K, He J, Zhang Q, Wang G, Uher C, Dravid V $P$ and Kanatzidis M G 2011 Strained endotaxial nanostructures with high thermoelectric figure of merit Nat. Chem. 3160

35. Androulakis J, Lin C-H, Kong H-J, Uher C, Wu C-I, Hogan T, Cook B A, Caillat T, Paraskevopoulos K M and Kanatzidis M G 2007 Spinodal decomposition and nucleation and growth as a means to bulk nanostructured thermoelectrics: enhanced performance in $\mathrm{Pb}_{1-\mathrm{x}} \mathrm{Sn}_{\mathrm{x}} \mathrm{Te}-\mathrm{PbS}$ J. Am. Chem. Soc. 129 9780

36. Zhang J, Wu D, He D, Feng D, Yin M, Qin X and He J 2017 Extraordinary thermoelectric performance realized in n-Type PbTe through multiphase nanostructure engineering Adv. Mater. 291703148 